Citation: S. Pinna (2020). I tornado negli USA: un'analisi dell'archivio dati (19502018). Bollettino della Società Geografica Italiana serie 14, 3(2): 3-9. doi: 10.36253/bsgi-1208

Copyright: @ 2020 S. Pinna. This is an open access, peer-reviewed article published by Firenze University Press (http://www.fupress.com/bsgi) and distributed under the terms of the Creative Commons Attribution License, which permits unrestricted use, distribution, and reproduction in any medium, provided the original author and source are credited.

Data Availability Statement: All relevant data are within the paper and its Supporting Information files.

Competing Interests: The Author(s) declare(s) no conflict of interest.

\section{I tornado negli USA: un'analisi dell'archivio dati (1950-2018)}

\author{
An analysis of the U.S. tornado data archive (1950-2018)
}

\author{
Sergio Pinna \\ Dipartimento Civiltà e Forme del Sapere, Università di Pisa, Italia \\ E-mail: sergio.pinna@unipi.it
}

\begin{abstract}
A statistical analysis of the data contained in the NOAA's Storm Prediction Center tornado archive (covering the period 1950-2018) has been carried out. The actual average values of the frequencies for the various intensity classes could be effectively provided for the period 1991-2018, because of some inhomogeneities of the archive due to variations in the methods and procedures of tornado reporting. The time series of the frequency of F2, F3, F4 and F5 events showed decreasing trends; this decline seems largely due to a significant reduction of the strongest events. This interpretation is supported by the decreasing trends of normalized economic losses and of number of victims.
\end{abstract}

Keywords: tornado, USA, Fujita intensity, frequency, time series.

Riassunto. È stata condotta un'analisi dei dati contenuti nell'archivio dei tornado dello Storm Prediction Center della NOAA. Questi dati coprono il periodo 1950-2018, ma, per il calcolo dei valori medi delle frequenze delle varie classi di intensità dei fenomeni, sono risultati utilizzabili solo quelli dell'intervallo 1991-2018, a causa di disomogeneità nell'archivio, dovute alle variazioni nei metodi e nelle procedure di osservazione. Le serie temporali della frequenza dei tornado F2 e superiori hanno mostrato dei trend decrescenti, che sono in buona parte dovuti a un'effettiva riduzione dell'incidenza degli eventi più forti. L'andamento delle perdite economiche normalizzate e quello del numero delle vittime danno sostegno a questa interpretazione.

Parole chiave: tornado, USA, intensità Fujita, frequenza, serie storiche.

\section{Introduzione}

In ragione del concorso di vari fattori geografici e meteorologici, il territorio degli Stati Uniti è quello sul quale si riscontra la più elevata frequenza di tornado di tutto il pianeta. Vista l'elevata pericolosità potenziale di queste manifestazioni atmosferiche, non sorprende quindi che sia proprio tale paese ad aver iniziato per primo i relativi studi sul fenomeno, con adeguate osser- 
vazioni sistematiche. L'archivio ${ }^{1}$ dell'SPC (Storm Prediction Center) della NOAA (National Oceanic and Atmospheric Administration) può così disporre di statistiche che risalgono agli anni Cinquanta dello scorso secolo, le uniche dalle quali è pertanto possibile ricavare delle serie storiche che consentano di valutare, pur con varie incertezze di cui si darà conto, l'evoluzione temporale dei tornado.

L'argomento assume oggi una notevole importanza anche nell'ambito del dibattito sul cambiamento climatico, con ovvio riferimento alla questione degli eventi estremi, un tema troppo spesso affrontato con una sorta di presupposti dogmatici e senza fare il dovuto ricorso a un'analisi diretta delle osservazioni sperimentali.

I contenuti del presente lavoro sono appunto dedicati ad alcune analisi sui dati (1950-2018) dell'archivio sopra citato, in merito al quale è però indispensabile fare una considerazione preliminare, dovuta alla presenza in esso di un problema, emerso durante la consultazione effettuata nel mese di ottobre 2020. In effetti, vi è un errore sistematico che comporta una duplicazione dei valori una volta ogni quattro anni, a partire dal 1956; ad esempio, 3622 è il numero totale indicato per l'anno 2004, mentre il dato reale è intorno a 1800 , come si può verificare nello studio di Verbout et al. (2007) e come appare anche in un grafico presente in una nota esplicativa ${ }^{2}$ del sito stesso dell'archivio. Sempre della NOAA è però possibile consultare una pagina web dedicata alla climatologia dei tornado $^{3}$, nella quale le statistiche essenziali sono fornite correttamente, cioè senza l'errore citato ${ }^{4}$.

In definitiva, l'uso combinato delle due fonti ha permesso una valida ricostruzione delle serie storiche delle frequenze, per le diverse classi di intensità dei fenomeni.

\section{Le frequenze medie annue}

I tornado sono catalogati secondo sei classi di intensità, in base alla scala Fujita (dal livello minimo F0 al massimo F5); dal 2007 tale scala è stata poi aggiornata secondo la cosiddetta Enhanced Fujita, sempre articolata in sei categorie, da EF0 a EF5. Gli esperti statunitensi non hanno comunque ritenuto necessario un riesame degli episodi antecedenti al 2007 secondo i nuovi criteri, per cui si deve assumere che, nella pratica, non vi sia una sostanziale soluzione di continuità nelle valutazioni

\footnotetext{
${ }^{1}$ https://www.spc.noaa.gov/climo/online/sp3

${ }^{2}$ https://www.spc.noaa.gov/efscale/

${ }^{3}$ https://www.ncdc.noaa.gov/climate-information/extreme-events/ustornado-climatology/trends

${ }^{4}$ L'incongruenza fra le due fonti è stata da me segnalata alla NOAA, senza però ottenere alcun riscontro.
}

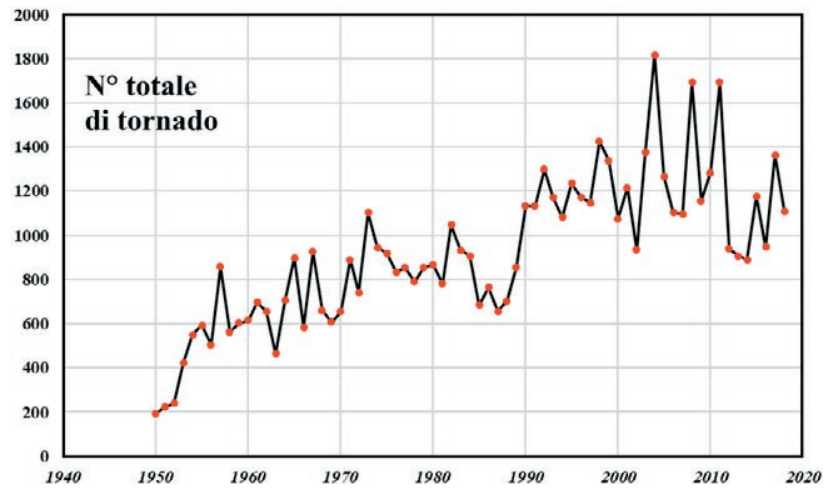

Figura 1. Landamento del numero totale annuo di tornado negli USA, come risulta dai dati registrati nell'archivio della NOAA.

degli eventi. Nel seguito della trattazione, per motivi di semplicità, la sigla $F$ verrà pertanto utilizzata per l'inquadramento, non solo dei tornado dal 1950 al 2006, ma pure di quelli successivi a questo periodo.

La figura 1 mostra l'andamento del numero annuo di tutti gli eventi censiti nell'archivio: è evidente un trend generale di aumento, determinato nello specifico da una crescita progressiva nel primo quarantennio di osservazioni, cui fanno seguito un brusco salto dei valori e poi una sostanziale stabilizzazione attorno a una media di poco superiore a 1200 . Gli incrementi evidenziati dal grafico ben poco hanno a che vedere con le oscillazioni del clima, ma sono in sostanza dovuti a un insieme di fattori non climatici che, nel corso del tempo, hanno aumentato la probabilità dell'individuazione delle manifestazioni più deboli (gli F1 e soprattutto gli F0), che inizialmente sfuggivano in buona parte alle rilevazioni.

È chiaro pertanto che il dataset disponibile contiene evidenti disomogeneità fra periodi diversi, per cui il calcolo di medie generali condurrebbe a valori mal rappresentativi della situazione reale; le statistiche che saranno allora fornite nel seguito del presente paragrafo sono relative all'intervallo 1991-2018.

Il numero medio annuo di tornado rilevati è 1215,3 con netta prevalenza di quelli deboli, rispetto ai significativi (Tab. 1 e Fig. 2).

Gli F0 costituiscono da soli oltre il $60 \%$ di tutti gli eventi riconosciuti ed è assai probabile che, nonostante

Tabella 1. Statistiche essenziali (1991-2018) dei tornado negli USA.

\begin{tabular}{lcccc}
\hline & F0 & F1 & F2/+ & Totale \\
\hline numero medio annuo & 733,8 & 348,7 & 132,8 & 1215,3 \\
deviazione standard & 150,8 & 96,1 & 42,5 & 232,9 \\
coefficiente di variazione (\%) & 20,6 & 27,6 & 32,0 & 19,2 \\
\hline
\end{tabular}




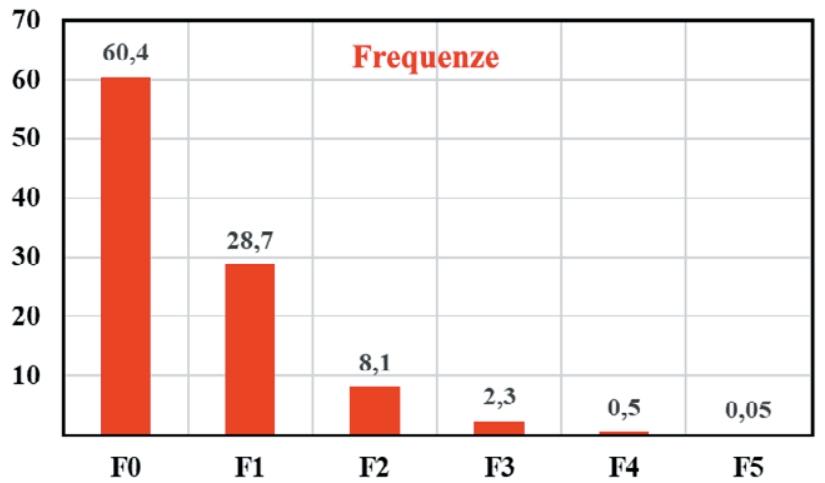

Figura 2. Le frequenze medie (\%) dei tornado, secondo le sei classi d'intensità.

i grandi progressi nella ricerca, un certo numero di essi sfugga ancora alle rilevazioni; gli F2 e superiori non arrivano nel loro complesso all'11\%. Ciò dimostra che i problemi sociali dovuti a questo fenomeno derivano in parte predominante da un numero estremamente limitato di episodi. Si tenga conto in proposito che tra il 1996 e il 2010 gli F0 ed F1 hanno prodotto il $21 \%$ dei danni totali, a fronte quindi del $79 \%$ causato da quelli di intensità maggiore.

\section{L'andamento temporale delle frequenze}

In ragione di quanto già detto a commento della figura 1, è ovvio che un quadro attendibile sullo sviluppo del fenomeno nel tempo possa emergere solo dall'esame delle serie storiche delle singole classi (Fig. 3).

Per quanto riguarda gli F0, l'aspetto più significativo è dato dal salto verificatosi attorno al 1990, con passaggio da una media di 336 nel quindicennio 1975-1989 a una superiore a 750 nel periodo successivo; la NOAA spiega che tale balzo è da addebitarsi all'attivazione della rete nazionale di radar Doppler, che ha consentito di identificare un numero di piccoli tornado molto maggiore rispetto al passato.

Il grafico degli F1 presenta varie analogie con quello degli F0, in particolare a proposito della evidente presenza di un salto che porta di colpo i valori da una media prossima a 220 in una prima fase, a una di 340 nella seconda; il salto è però posizionato in questo caso nel 1970, quindi un ventennio prima di quanto riscontrato per gli F0. Senza dubbio la causa è da ricercarsi ancora in qualche importante cambiamento avvenuto nelle modalità di indagine e di acquisizione dei dati; tuttavia nelle note del sito della NOAA non vi è spiegazione della ragione specifica. Incuriosisce poi la situazione degli anni più recenti, con alcuni valori molto elevati per gli $\mathrm{F} 1$, a fronte di una riduzione di quelli degli F0; è possibile che l'introduzione dei criteri della scala Enhanced Fujita abbia mutato la valutazione di certi tornado deboli, ma ciò, al momento, è una pura ipotesi.

Passando ai tornado "significativi" (appartenenti cioè alle classi F2 e superiori), il quadro generale delle serie si inverte, visto che le frequenze registrate tendono palesemente a diminuire; la conferma è data dal calcolo dei trend lineari ${ }^{5}$, tutti negativi (Tab. 2).

Soltanto per la classe F5 è risultato un insufficiente livello di confidenza, dovuto al limitatissimo numero di questi episodi di massima violenza, che rende la serie temporale poco significativa del punto di vista statistico; si tenga infatti presente che in tutto il periodo di osservazione ne sono stati censiti soltanto 59 , con 39 annate prive di tali manifestazioni. Calcolando in base ai coefficienti angolari delle rette di regressione, si evince che le riduzioni registrate sarebbero davvero consistenti: tra l'inizio e la fine dell'intervallo temporale considerato, ammonterebbero infatti al 62, 53, 67 e 48\%, rispettivamente per F2, F3, F4 ed F5. Questi dati implicano che si tratti di una variazione dovuta a un mutamento delle condizioni atmosferiche medie oppure ad altro?

Brooks e Craven (2002) hanno risposto a questa domanda chiamando in causa dei cambiamenti avvenuti nelle modalità di registrazione dei tornado: il passaggio di competenze nel 1973 dal programma climatologico federale al National Weather Service e l'adozione della scala Fujita nel 1975. L'interpretazione fornita è sicuramente valida ma, a mio giudizio, tutt'altro che esaustiva per i motivi di seguito espressi.

La riduzione del numero di tornado significativi pare troppo ampia per poter essere prodotta dalle sole cause prima citate; è un problema, in qualche modo, riconosciuto anche dagli stessi Autori.

L'applicazione alle serie di frequenza degli F2 ed F3 dei test di omogeneità di Pettitt e di Buishand fa individuare in esse la presenza di un change point, con confidenza del 99,9\%; per entrambe, esso è però collocato nel 1984, quindi spostato di vari anni rispetto alle modifiche segnalate da Brooks e Craven.

Simmons et al. (2013) hanno condotto una ricerca sulle conseguenze economiche dei tornado negli USA, nel periodo 1950-2011. Normalizzando i valori rispetto al 2011, in ragione dei cambiamenti nell'economia e nel territorio, hanno osservato un decremento nel tempo così consistente da far loro ritenere che esso sia in buona

\footnotetext{
${ }^{5}$ Seguendo quanto fatto dalla stessa NOAA, le serie sono studiate a partire dal 1954, in ragione delle carenze di dati riscontrabili per i primi anni di rilevazione.
} 

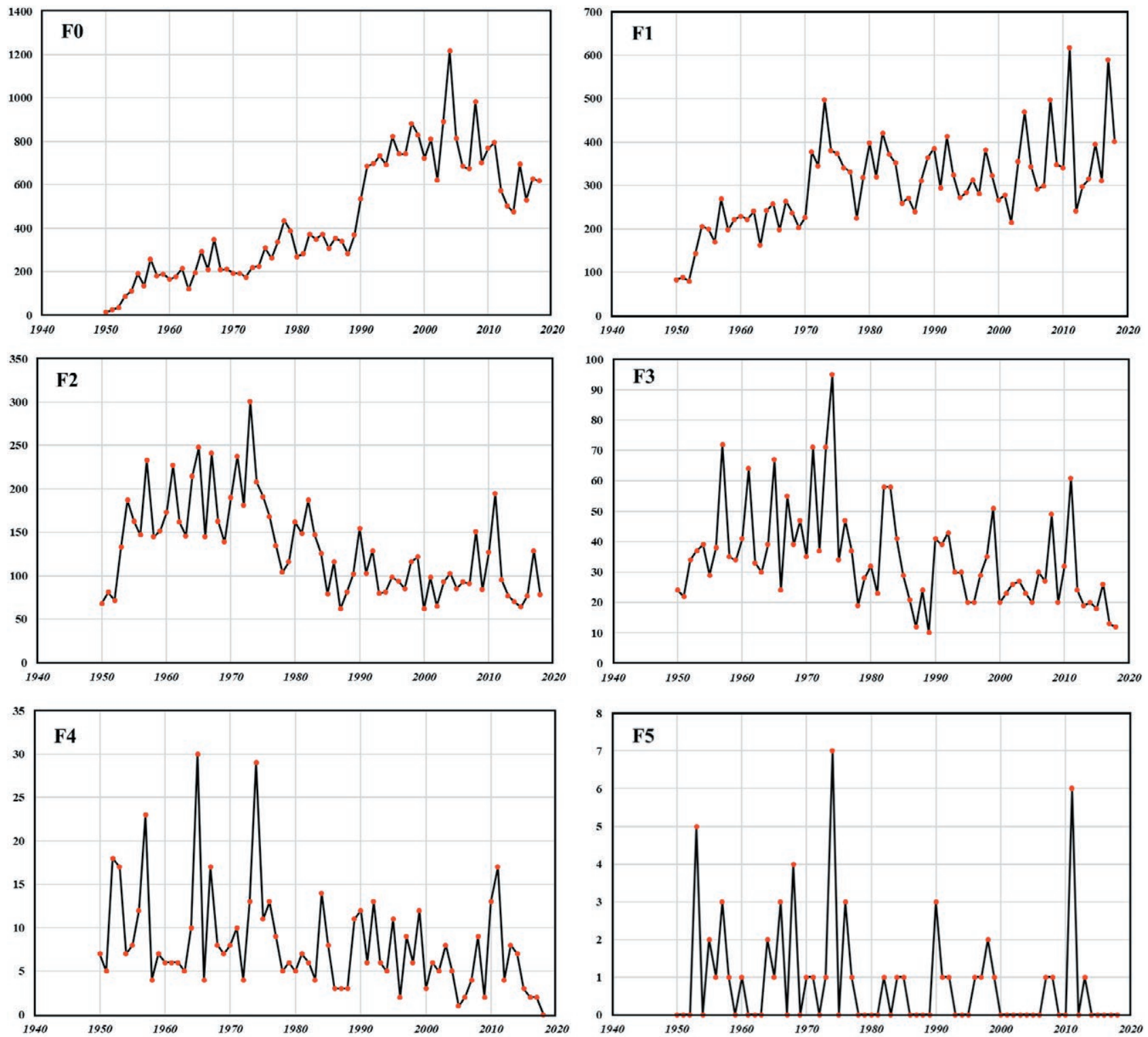

Figura 3. L'andamento del numero annuo di tornado ricadenti nelle diverse classi di intensità.

parte il segnale che - pur considerando tutti i possibili effetti dovuti alle modifiche nelle procedure di segnala-

Tabella 2. Parametri dei trend lineari per le classi degli eventi significativi.

\begin{tabular}{cccc}
\hline & Coeff. Ang. & $\mathrm{R}^{2}$ & Confidenza (\%) \\
\hline F2 & $-1,923$ & 0,450 & 99,9 \\
F3 & $-0,404$ & 0,203 & 99,9 \\
F4 & $-0,100$ & 0,105 & 99 \\
F5 & $-0,013$ & 0,033 & n.s. \\
\hline
\end{tabular}

zione e registrazione degli eventi - conferma un'effettiva diminuzione dell'incidenza dei tornado di forte intensità. Sottoponendo ai test di omogeneità la serie normalizzata in base ai cambiamenti nel reddito e nella densità delle abitazioni, vi si riscontra un change point (confidenza 99,9\%) con posizione più probabile proprio nel 1984, cioè in analogia a quello delle serie delle frequenze degli F2 ed F3; tale change point segna una riduzione delle perdite di circa il $46 \%$ per il periodo $1985-2011$ rispetto agli anni antecedenti (Fig. 4).

In definitiva, allo stato attuale delle conoscenze, sembra evidente come i trend negativi di cui alla tabel- 

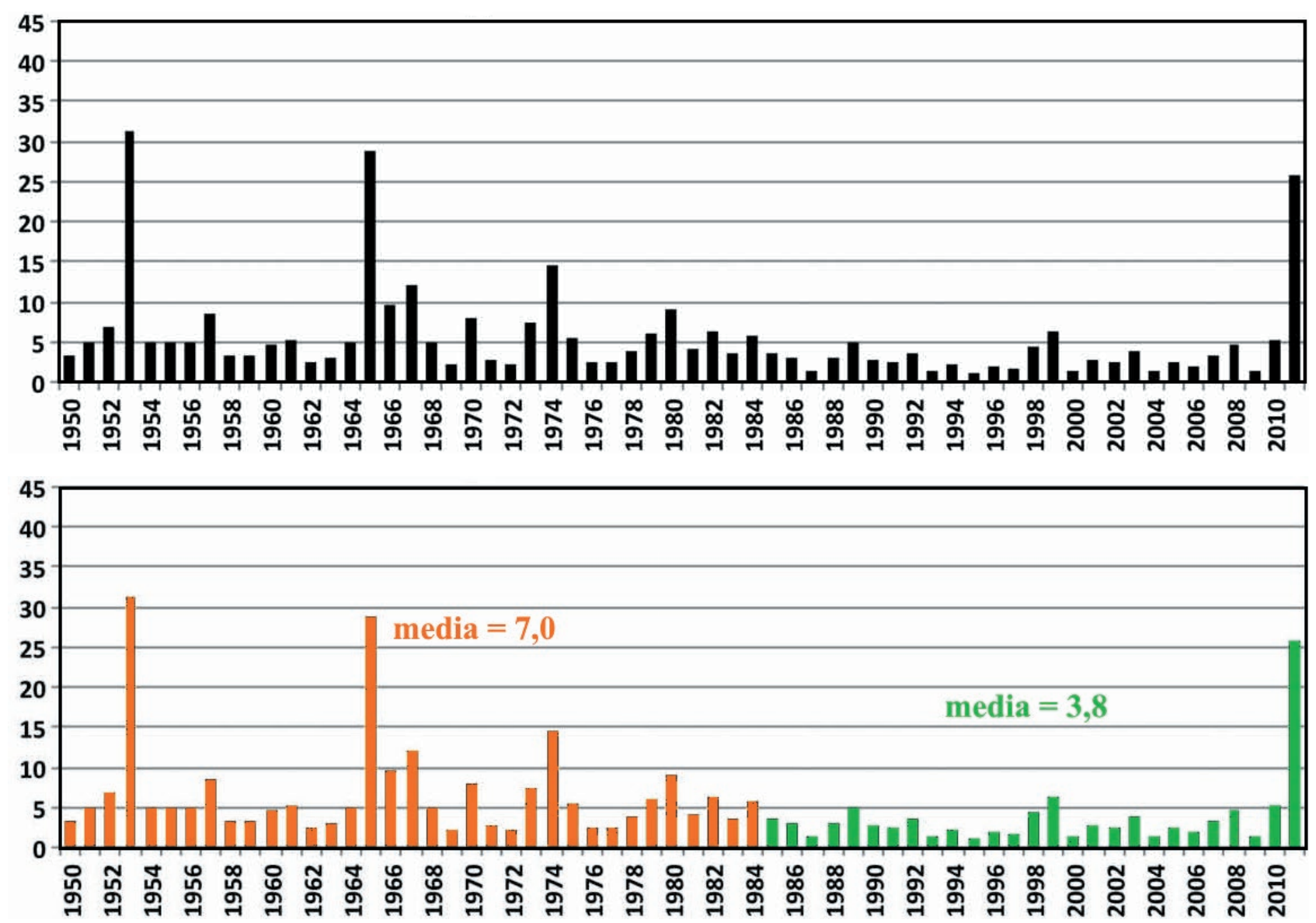

Figura 4. Perdite economiche negli USA, in miliardi di dollari, per i tornado. In alto: il grafico (c) da pag. 140 di Simmons et al. (2013). In basso: la stessa figura con i colori che evidenziano l'ampia differenza fra le due parti della serie, di cui nel testo.

la 1 dipendano da due cause congiunte: a) le sovrastime nella classificazione dei tornado antecedenti alla metà degli anni '70; b) una considerevole riduzione della frequenza degli episodi intensi, a partire dal decennio successivo.

\section{Le statistiche delle vittime}

Che le manifestazioni tornadiche costituiscano un serio problema sociale per gli Stati Uniti è dimostrato, oltre che dalle consistenti perdite economiche, anche dal contributo pagato in termini di vite umane; dal 1950 al 2018 le vittime sono state infatti 7047, per una media quindi di 102 ogni anno.

Un enorme numero di eventi, come già visto in precedenza, è di debole entità e procura una piccola quantità di vittime; a F0 ed F1 compete infatti solo il 6\% circa del totale (Fig. 5). Sono gli F3 ad avere la quota maggiore
(33\%), in quanto tale classe è caratterizzata da una intensità già assai elevata e da una frequenza ancora abbastanza alta.

Rapportando, per ciascuna classe Fujita, il numero totale delle vittime a quello dei tornado, si ottiene un valore che ne quantifica il livello di pericolosità, in termini di perdita di vite umane: se il verificarsi di un F0 o di un F1 implica una probabilità estremamente bassa di avere delle vittime, il dato sale molto nelle categorie maggiori, arrivando a 28,6 per un F5 (Tab. 3).

Per quanto concerne l'andamento temporale del numero annuo di vittime, si constata una riduzione evidente, come ben chiarito dal trend lineare negativo (confidenza del 94\%) della figura 6. Riferendosi alle due fasi individuate nei precedenti paragrafi, si calcola una media di 125,8 per il $1950-1984$ e una di 77,7 per gli anni successivi; cioè un calo del rischio di circa il $38 \%$.

È logico porsi allora la domanda se questo decremento possa essere interpretato come un ulteriore 


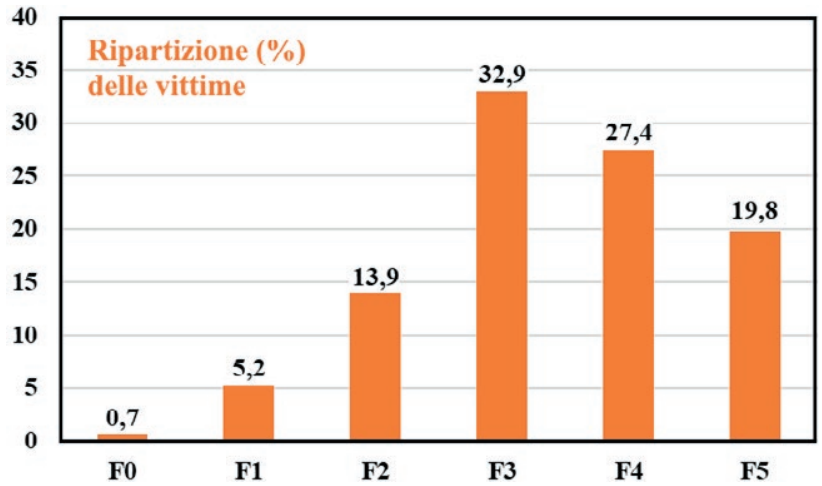

Figura 5. La ripartizione media (1991-2018) delle vittime, secondo le classi di intensità.

Tabella 3. Statistiche delle vittime (1991-2018), per le classi dei tornado.

\begin{tabular}{lcccccc}
\hline & F0 & F1 & F2 & F3 & F4 & F5 \\
\hline vittime & 16 & 121 & 321 & 760 & 632 & 458 \\
$\mathrm{~N}^{\circ}$ tornado & 20547 & 9763 & 2745 & 787 & 171 & 16 \\
vittime $/ \mathrm{N}^{\circ}$ & 0,001 & 0,01 & 0,12 & 0,97 & 3,70 & 28,62 \\
\hline
\end{tabular}

segnale di calo dell'incidenza dei tornado più forti. Per poter dare una risposta precisa, sarebbe necessario disporre di una valutazione adeguata delle variazioni nella vulnerabilità, cosa davvero non semplice; tuttavia si possono fare le seguenti considerazioni. Da un lato, il progresso nelle previsioni meteorologiche e lo sviluppo di sistemi di allerta sempre più efficaci hanno fornito maggiore protezione alle persone; dall'altro però bisogna tenere presente che la popolazione potenzialmente esposta è più che raddoppiata (gli USA sono infatti passati dai circa 158 milioni di abitanti del 1950 ai quasi 330 attuali).

In definitiva sembra decisamente ragionevole ritenere - pur con le dovute cautele - che la vulnerabilità non si sia nel complesso ridotta in modo sensibile, e perciò che il calo del rischio verificatosi possa essere ascritto, almeno in buona parte, a una diminuzione della pericolosità del fenomeno.

\section{Brevi riflessioni conclusive}

Le verifiche condotte sui dati dell'archivio statunitense hanno permesso di constatare che l'incidenza dei forti tornado negli ultimi 30-35 anni si è notevolmente ridotta, rispetto a quanto avveniva nei decenni precedenti. Questa osservazione - considerando che l'inte-

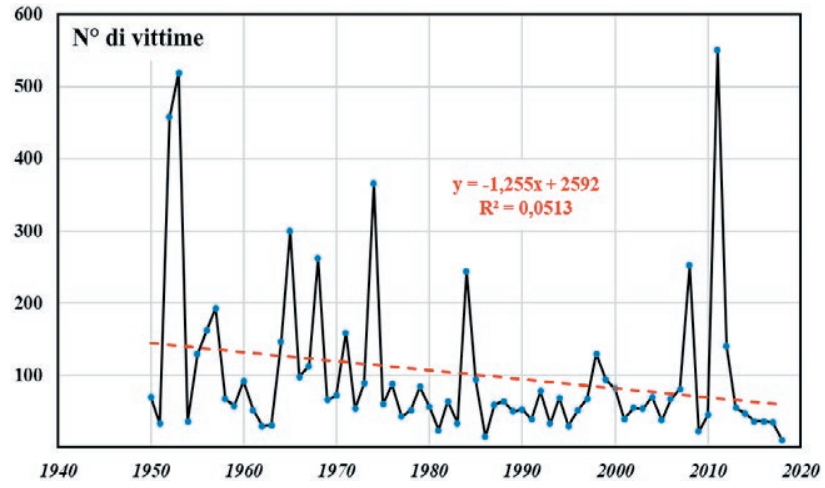

Figura 6. La serie storica del numero annuo di vittime; in rosso il trend lineare e la relativa equazione.

ra serie storica ha una lunghezza di 70 anni - non può essere utilizzata per proiezioni sugli sviluppi futuri, ma è di sicuro interesse climatologico, perché costituisce un esempio di situazione opposta alle teorie oggi imperanti, che indicano una correlazione diretta fra riscaldamento globale e aumento degli eventi meteorologici estremi.

Purtroppo, nel dibattito sul cambiamento climatico, si nota che la divulgazione di informazioni non in linea con certe posizioni ufficiali incontra serie difficoltà, cosicché l'opinione pubblica viene spesso orientata in modo non corretto; anche i tornado negli USA ne hanno recentemente fornito un esempio.

Dopo il 2011, quando si verificarono molti tornado violenti (con ben sei F5) che portarono al record di 550 vittime, determinando una larga attenzione mediatica su questi temi, si è avuta una serie di annate caratterizzate da un ritorno della pericolosità su livelli decisamente bassi; su tale fatto è però calato il silenzio, favorendo così nelle persone il diffondersi di una visione molto lontana dalla realtà. A riprova di ciò, è infatti da notare che, dopo l'ampia enfasi con la quale erano stati comunicati gli eventi disastrosi del 2011, nessuno esperto ha fatto nemmeno un cenno sul 2018, l'annata di record negativo assoluto di tutta la serie storica: soltanto 10 vittime e neanche un evento di intensità superiore alla classe F3. Una vera eccezionalità climatica del tutto ignorata dal sistema dell'informazione.

\section{Riferimenti bibliografici}

Brooks E., Craven J. P. (2002). A database of proximity soundings for significant severe thunderstorms, 19571993. Preprints, 21st Conference on Severe Local Storms, San Antonio, TX, American Meteorological Society, 639642. 
Edwards R., LaDue J. G., Ferree J. T., Scharfenberg K., Maier C., Coulbourne W. L. (2013). Tornado Intensity Estimation. Past, Present, and Future. Bulletin of the American Meteorological Society, 94 (5), 641-653.

Simmons K. M., Sutter D., Pielke R. (2013). Normalized tornado damage in the United States: 1950-2011. Environmental Hazards, 12 (2), 132-147, http://dx.doi.org/10. 1080/17477891.2012.738642

Verbout S. M., Schultz D. M., Leslie L. M., Brooks H. E., Karoly D. J., Elmore K. L. (2007). Tornado outbreaks associated with landfalling hurricanes in the north Atlantic Basin: 1954-2004. Meteorology and Atmospheric Physics, 97, 255-271.

Verbout S. M., Schultz D. M., Leslie L. M., Brooks H. E. (2013). Evolution of the U.S. Tornado Database: 19542003. Weather and Forecasting. 21, 86-93. 